Journal of Mathematical Physics, Analysis, Geometry

2017 , vol. 13 , No. 4 , pp. $353-363$

doi:10.15407/mag13.04.353

\title{
On Compact Super Quasi-Einstein Warped Product with Nonpositive Scalar Curvature
}

\author{
Sampa Pahan ${ }^{1}$, Buddhadev Pal ${ }^{2}$, and Arindam \\ Bhattacharyya $^{3}$ \\ ${ }^{1,3}$ Jadavpur University, Department of Mathematics \\ Kolkata 700032, India \\ E-mail: ${ }^{1}$ sampapahan25@gmail.com \\ ${ }^{3}$ bhattachar1968@yahoo.co.in \\ ${ }^{2}$ Banaras Hindu University, Institute of Science, Department of Mathematics \\ Varanasi 221005, India \\ E-mail: ${ }^{2}$ pal.buddha@gmail.com
}

Received August 24, 2015; revised September 27, 2016

This note deals with super quasi-Einstein warped product spaces. Here we establish that if $M$ is a super quasi-Einstein warped product space with nonpositive scalar curvature and compact base, then $M$ is simply a Riemannian product space. Next we give an example of super quasi-Einstein space-time. In the last section a warped product is defined on it.

Key words: Einstein manifold, super quasi-Einstein manifold, Ricci tensor, Hessian tensor, warped product, warping function.

Mathematical Subject Classification 2010: 53C20, 53B20.

\section{Introduction}

An $n$-dimensional $(n>2)$ Riemannian manifold is Einstein if its Ricci tensor $S$ of type $(0,2)$ is of the form $S=\alpha g$, where $\alpha$ is a smooth function, which turns into $S=\frac{r}{n} g, r$ being the scalar curvature of the manifold. The above equation is also called the Einstein metric condition [1]. Let $\left(M^{n}, g\right), n>2$, be a Riemannian manifold and $U_{S}=\left\{x \in M: S \neq \frac{r}{n} g\right.$ at $\left.x\right\}$, then the manifold $\left(M^{n}, g\right)$ is said to be quasi-Einstein manifold $[5,7]$ if on $U_{S} \subset M$ we have

$$
S-\alpha g=\beta A \otimes A,
$$

The first author is supported by UGC JRF of India 23/06/2013(i)EU-V.

(C) Sampa Pahan, Buddhadev Pal, and Arindam Bhattacharyya, 2017 
where $A$ is a 1 -form on $U_{S}$, and $\alpha$ and $\beta$ are some functions on $U_{S}$. It is clear that the 1 -form $A$, as well as the function $\beta$, is nonzero at every point on $U_{S}$. From the above definition, it follows that every Einstein manifold is quasi-Einstein. In particular, every Ricci-flat manifold (e.g., Schwarzchild space-time) is quasiEinstein. The scalars $\alpha, \beta$ are known as the associated scalars of the manifold. Also, the 1-form $A$ is called the associated 1-form of the manifold defined by $g(X, \rho)=A(X)$ for any vector field $X, \rho$ being a unit vector field, called the generator of the manifold. Such an $n$-dimensional quasi-Einstein manifold is denoted by $(Q E)_{n}$.

M.C. Chaki introduced the super quasi-Einstein manifold in [4], denoted by $S(Q E)_{n}$, where the Ricci tensor $S$ of type $(0,2)$, which is not identically zero, satisfies the condition

$$
\begin{aligned}
S(X, Y)=\alpha g(X, Y) & +\beta A(X) A(Y) \\
& +\gamma[A(X) B(Y)+A(Y) B(X)]+\delta D(X, Y),
\end{aligned}
$$

where $\alpha, \beta, \gamma, \delta$ are scalar functions such that $\beta, \gamma, \delta$ are nonzero and $A, B$ are two nonzero 1-forms such that $g(X, U)=A(X)$ and $g(X, V)=B(X), U, V$ being unit vectors which are orthogonal, i. e., $g(U, V)=0$ and $D$ is a symmetric $(0,2)$ tensor with zero trace which satisfies the condition $D(X, U)=0, \forall X \in \chi(M)$.

Here $\alpha, \beta, \gamma, \delta$ are called the associated scalars, and $A, B$ are called the associated main and auxiliary 1-forms, respectively, $U, V$ are the main and auxiliary generators, and $D$ is called the associated tensor of the manifold.

The notion of a warped product generalizes that of a surface of revolution. It was introduced in [3] for studying manifolds of negative curvature. Let $\left(B, g_{B}\right)$ and $\left(F, g_{F}\right)$ be two Riemannian manifolds with $\operatorname{dim} B=m>0, \operatorname{dim} F=k>0$ and $f: B \rightarrow(0, \infty), f \in C^{\infty}(B)$. Consider the product manifold $B \times F$ with its projections $\pi: B \times F \rightarrow B$ and $\sigma: B \times F \rightarrow F$. The warped product $B \times{ }_{f} F$ is the manifold $B \times F$ with the Riemannian structure such that $\|X\|^{2}=\left\|\pi^{*}(X)\right\|^{2}+$ $f^{2}(\pi(p))\left\|\sigma^{*}(X)\right\|^{2}$ for any vector field $X$ on $M$. Thus we have $g_{M}=g_{B}+f^{2} g_{F}$ holds on $M$. Here $B$ is called the base of $M$ and $F$ the fiber. The function $f$ is called the warping function of the warped product [10]. We will denote by $\operatorname{Ric}_{M}, \operatorname{Ric}_{B}, \operatorname{Ric}_{F}$, and $H^{f}$ the Ricci curvature of $\mathrm{M}$, the lifts to $M$ of the Ricci curvatures of $B$ and $F$, and the Hessian of $f$, respectively. A Riemannian manifold is said to be super quasi-Einstein if its Ricci tensor is proportional to the metric, that is,

$$
\begin{aligned}
\operatorname{Ric}_{M}=\alpha g_{M}(X, Y) & +\beta A(X) A(Y) \\
& +\gamma[A(X) B(Y)+A(Y) B(X)]+\delta D(X, Y) .
\end{aligned}
$$

By $\tau_{M}, \tau_{B}$ and $\tau_{F}$, we will understand the scalar curvatures of $M, B$ and $F$, that is, $\tau_{M}=\operatorname{Tr}\left(\operatorname{Ric}_{M}\right), \tau_{B}=\operatorname{Tr}\left(\operatorname{Ric}_{B}\right)$ and $\tau_{F}=\operatorname{Tr}\left(\operatorname{Ric}_{F}\right)$. Therefore we have the followings [10]: 
Proposition 1.1. The Ricci curvature Ric of the warped product $M=$ $B \times{ }_{f} F$ with $k=\operatorname{dim} F$ satisfies

(1) $\operatorname{Ric}(X, Y)=\operatorname{Ric}_{B}(X, Y)-\frac{k}{f} H^{f}(X, Y)$,

(2) $\operatorname{Ric}(X, V)=0$,

(3) $\operatorname{Ric}(V, W)=\operatorname{Ric}_{F}(V, W)-g(V, W) f^{\#}, f^{\#}=\frac{-\Delta f}{f}+\frac{k-1}{f^{2}}|\nabla f|^{2}$

for any horizontal vectors $X, Y$ (that is $X, Y \in \tau(T B)$ ) and any vertical vectors $V, W$ (that is $V, W \in \tau(T F)$ ), where $H^{f}$ and $\Delta f$ denote the Hessian of $f$ and the Laplacian of $f$ given by $\Delta f=-\operatorname{tr}\left(H^{f}\right)$, respectively.

Proposition 1.2. Let $M=B \times_{f} F$ be a warped product manifold. Then the scalar curvature of $M$ is given by

$$
\tau_{M}=\tau_{B}+\frac{\tau_{F}}{f^{2}}+2 k \frac{\Delta f}{f}-k(k-1) \frac{|\nabla f|^{2}}{f^{2}} .
$$

From the above Proposition 1.1 we get the following theorem.

Theorem 1.1. Let $M=B \times{ }_{f} F$ be a warped product manifold which is also a super quasi-Einstein manifold. Then the following conditions hold.

i) When $U, V$ are orthogonal and tangent to the base $B$, then the Ricci tensors of $B$ and $F$ satisfy the following conditions:

a) $\operatorname{Ric}_{B}(X, Y)=\alpha g_{B}(X, Y)+\beta g_{B}(X, U) g_{B}(Y, U)$

$$
\begin{aligned}
& +\gamma\left[g_{B}(X, U) g_{B}(Y, V)+g_{B}(Y, U) g_{B}(X, V)\right] \\
& +\delta D_{B}(X, Y)+\frac{k}{f} H^{f}(X, Y),
\end{aligned}
$$

b) $\operatorname{Ric}_{F}(X, Y)=g_{F}(X, Y)\left[\alpha f^{2}-f \Delta f+(k-1)|\nabla f|^{2}\right]+\delta D_{F}(X, Y)$;

ii) When $U, V$ are orthogonal and tangent to the fibre $F$, then the Ricci tensors of $B$ and $F$ satisfy the following conditions:

a) $\operatorname{Ric}_{B}(X, Y)=\alpha g_{B}(X, Y)+\frac{k}{f} H^{f}(X, Y)+\delta D_{B}(X, Y)$,

b) $\operatorname{Ric}_{F}(X, Y)=g_{F}(X, Y)\left[\alpha f^{2}-f \Delta f+(k-1)|\nabla f|^{2}\right]$

$$
\begin{aligned}
& +\beta f^{4} g_{F}(X, U) g_{F}(Y, U)+\gamma f^{4}\left[g_{F}(X, U) g_{F}(Y, V)\right. \\
& \left.+g_{F}(Y, U) g_{F}(X, V)\right]+\delta D_{F}(X, Y) .
\end{aligned}
$$

Corollary 1.1. Taking the traces of Theorem 1.1, we get the scalar curvature of $M, B$ and $F$ of two different cases. 
i) $\tau_{M}=\alpha(m+k)+\beta, \tau_{B}=\alpha m-k \frac{\Delta f}{f}+\beta, \tau_{F}=k\left[\alpha f^{2}-f \Delta f+(k-1)|\nabla f|^{2}\right]$.

ii) $\tau_{M}=\alpha(m+k)+\beta, \tau_{B}=\alpha m-k \frac{\Delta f}{f}, \tau_{F}=k\left[\alpha f^{2}-f \Delta f+(k-1)|\nabla f|^{2}\right]+$ $\beta f^{4}$.

The proves of Theorem 1.1 and Corollary 1.1 follow similarly to Theorem 2.1 from the paper [12]. We also have the following propositions from [2,10], where the expression of Ricci curvature of a warped product space was obtained.

Many authors, like M.C. Chaki [4], C. Özgür [11], etc., have studied super quasi-Einstein manifolds. In [6], D. Dumitru gave a characterization of the warped product on quasi-Einstein manifold and B. Pal, A. Bhattacharyya studied a characterization of the warped product on mixed super quasi-Einstein manifold in [12]. In [9], D. Kim discussed about a compact Einstein warped space with nonpositive scalar curvature. Motivated by the above papers, in this work we study super quasi-Einstein warped product spaces with nonpositive scalar curvature. Also, we establish the four-dimensional example of super quasi-Einstein space-time, and in the last section we give the example of a warped product on it.

\section{Super Quasi-Einstein Warped Product Spaces with Nonpositive Scalar Curvature}

From Proposition 1.1, we get the following result where equation (1.2) becomes

Result 2.1. When $U, V$ are orthogonal and tangent to the base $B$, the warped product $M=B \times_{f} F$ is a super quasi-Einstein manifold with

$\operatorname{Ric}_{M}(X, Y)=\alpha g_{M}(X, Y)+\beta A(X) A(Y)+\gamma[A(X) B(Y)+A(Y) B(X)]+\delta D(X, Y)$, where $D(X, Y)=g(l X, Y), l$ is a symmetric endomorphism if and only if

(2.a) $\operatorname{Ric}_{B}(X, Y)=\alpha g_{B}(X, Y)+\beta g_{B}(X, U) g_{B}(Y, U)$

$$
\begin{aligned}
& +\gamma\left[g_{B}(X, U) g_{B}(Y, V)+g_{B}(Y, U) g_{B}(X, V)\right] \\
& +\delta D_{B}(X, Y)+\frac{k}{f} H^{f}(X, Y),
\end{aligned}
$$

(2.b) $\operatorname{Ric}_{F}(X, Y)=\mu g_{F}(X, Y)+\delta D_{F}(X, Y)$,

(2.c) $\mu=\left[\alpha f^{2}-f \Delta f+(k-1)|\nabla f|^{2}\right]$.

Now, we state a lemma whose detailed proof is given in [9]. 
Lemma 2.1. Let $f$ be a smooth function on a Riemannian manifold $B$, then for any vector $X$, the divergence of the Hessian tensor $H^{f}$ satisfies

$$
\operatorname{div}\left(H^{f}\right)(X)=\operatorname{Ric}(\nabla f, X)-\Delta(d f)(X),
$$

where $\Delta=d \delta+\delta d$ denotes the Laplacian on $B$ acting on differential forms.

Now we prove the following proposition.

Proposition 2.1. Let $\left(B^{m}, g_{B}\right)$ be a compact Riemannian manifold of dimension $m \geq 2$. Suppose that $f$ is a nonconstant smooth function on $B$ satisfying (2.a) for a constant $\alpha \in R$ and a natural number $k \in N$, and if the condition

$$
\begin{aligned}
\beta g_{B}(X, U) g_{B}(\nabla f, U) & +\gamma\left[g_{B}(X, U) g_{B}(\nabla f, V)\right. \\
& \left.+g_{B}(\nabla f, U) g_{B}(X, V)\right]+g_{B}(l X, \nabla f)=0
\end{aligned}
$$

holds, then $f$ satisfies (2.c) for a constant $\mu \in R$. Hence, for a compact Riemannian manifold $F$ with $\operatorname{Ric}_{F}(X, Y)=\mu g_{F}(X, Y)+\delta D_{F}(X, Y)$, we can make a compact super quasi-Einstein warped product space $M=B \times_{f} F$ with

$$
\begin{aligned}
\operatorname{Ric}_{M}(X, Y)=\alpha g_{M}(X, Y) & +\beta A(X) A(Y) \\
& +\gamma[A(X) B(Y)+A(Y) B(X)]+\delta D(X, Y),
\end{aligned}
$$

where $D(X, Y)=g(l X, Y), l$ is a symmetric endomorphism when $U, V$ are orthogonal and tangent to the base $B$.

Proof. By taking the trace of both sides of (2.a), we have

$$
S=\alpha m-k \frac{\Delta f}{f}+\beta,
$$

where $S$ denotes the scalar curvature of $B$ given by $\operatorname{tr}(\mathrm{Ric})$. Note that the second Bianchi identity implies (see [10])

$$
d S=2 \operatorname{div}(\text { Ric }) .
$$

From equations (2.2) and (2.3), we obtain

$$
\operatorname{div} \operatorname{Ric}(X)=\frac{k}{2 f^{2}}\{\Delta f d f-f d(\Delta f)(X)\}
$$

On the other hand, by the definition, we have

$$
\operatorname{div}\left(\frac{1}{f} H^{f}\right)(X)=\sum_{i}\left(D_{E_{i}}\left(\frac{1}{f} H^{f}\right)\right)\left(E_{i}, X\right)
$$




$$
=-\frac{1}{f^{2}} H^{f}(\nabla f, X)+\frac{1}{f} \operatorname{div} H^{f}(X)
$$

for any vector field $X$ and an orthonormal frame $E_{1}, E_{2}, \ldots, E_{m}$ of $B$. Since $H^{f}(\nabla f, X)=\left(D_{X} d f\right)(\nabla f)=\frac{1}{2} d\left(|\nabla f|^{2}\right)(X)$, the last equation becomes

$$
\operatorname{div}\left(\frac{1}{f} H^{f}\right)(X)=-\frac{1}{2 f^{2}} d\left(|\nabla f|^{2}\right)(X)+\frac{1}{f} \operatorname{div} H^{f}(X)
$$

for a vector field $X$ on $B$. Hence, from (2.a) and (2.1), it follows that

$$
\begin{aligned}
\operatorname{div}\left(\frac{1}{f} H^{f}\right)(X)=\frac{1}{2 f^{2}} & \left\{(k-1) d\left(|\nabla f|^{2}\right)-2 f d(\Delta f)+2 \alpha f d f\right\} \\
& +\frac{1}{f} \beta g_{B}(X, U) g_{B}(\nabla f, U) \\
& +\frac{1}{f} \gamma\left[g_{B}(X, U) g_{B}(\nabla f, V)+g_{B}(\nabla f, U) g_{B}(X, V)\right] \\
& +\frac{1}{f} \delta D_{B}(X, \nabla f) .
\end{aligned}
$$

But, (2.a) gives $\operatorname{div} \operatorname{Ric}_{B}=\operatorname{div}\left(\frac{k}{f} H^{f}\right)+\operatorname{div} D_{B}$. Therefore, (2.4) and (2.5) imply that $d\left(-f \Delta f+(k-1)|\nabla f|^{2}+\alpha f^{2}\right)=0$, that is, $-f \Delta f+(k-1)|\nabla f|^{2}+\alpha f^{2}=$ $\mu$ for some constant $\mu$. Thus the first part of the proposition is proved. For a compact Riemannian manifold $\left(F, g_{F}\right)$ of dimension $k$ with $\operatorname{Ric}_{F}=\mu g_{F}+\delta D_{F}$, we can construct a compact super quasi-Einstein warped product $M=B \times{ }_{f} F$ by the sufficiencies of Result 2.1 .

In a similar way, we get the following result and proposition when $U, V$ are orthogonal and tangent to the fiber $F$.

Result 2.2. When $U, V$ are orthogonal and tangent to the fiber $F$, the warped product $M=B \times_{f} F$ is a super quasi-Einstein manifold with $\operatorname{Ric}_{M}(X, Y)=$ $\alpha g_{M}(X, Y)+\beta A(X) A(Y)+\gamma[A(X) B(Y)+A(Y) B(X)]+\delta D(X, Y)$, where $D(X, Y)=g(l X, Y), l$ is a symmetric endomorphism. if and only if

$$
\begin{aligned}
& \text { (2.d) } \operatorname{Ric}_{B}(X, Y)=\alpha g_{B}(X, Y)+\frac{k}{f} H^{f}(X, Y)+\delta D_{B}(X, Y), \\
& \begin{array}{c}
(2 . \mathrm{e}) \operatorname{Ric}_{F}(X, Y)=g_{F}(X, Y)\left[\alpha f^{2}-f \Delta f+(k-1)|\nabla f|^{2}\right] \\
\quad+\beta f^{4} g_{F}(X, U) g_{F}(X, U) \\
\quad+\gamma f^{4}\left[g_{F}(X, U) g_{F}(Y, V)+g_{F}(Y, U) g_{F}(X, V)\right]+\delta D_{F}(X, Y), \\
\text { (2.f) } \mu=\left[\alpha f^{2}-f \Delta f+(k-1)|\nabla f|^{2}\right] .
\end{array}
\end{aligned}
$$


Proposition 2.2. Let $\left(B^{m}, g_{B}\right)$ be a compact Riemannian manifold of dimension $m \geq 2$. Suppose that $f$ is a nonconstant smooth function on $B$ satisfying (2.d) for a constant $\alpha \in R$ and a natural number $k \in N$, and if the condition $\delta g_{B}(l X, \nabla f)=0$ holds, then $f$ satisfies (2.f) for a constant $\mu \in R$. Hence, for a compact super quasi-Einstein manifold $F$ with

$$
\begin{aligned}
\operatorname{Ric}_{F}(X, Y)= & g_{F}(X, Y)\left[\alpha f^{2}-f \Delta f+(k-1)|\nabla f|^{2}+\beta f^{4} g_{F}(X, U) g_{F}(Y, U)\right. \\
& +\gamma f^{4}\left[g_{F}(X, U) g_{F}(Y, V)+g_{F}(Y, U) g_{F}(X, V)\right]+\delta D_{F}(X, Y),
\end{aligned}
$$

we can make a compact super quasi-Einstein warped product space $M=B \times_{f} F$ with

$$
\begin{aligned}
\operatorname{Ric}_{M}(X, Y)=\alpha g_{M}(X, Y) & +\beta A(X) A(Y) \\
& +\gamma[A(X) B(Y)+A(Y) B(X)]+\delta D(X, Y),
\end{aligned}
$$

where $D(X, Y)=g(l X, Y), l$ is a symmetric endomorphism when $U, V$ are orthogonal and tangent to the fiber $F$.

Proof. By taking the trace of both sides of (2.d), we have

$$
S=\alpha m-k \frac{\Delta f}{f}
$$

where $S$ denotes the scalar curvature of $B$ given by $\operatorname{tr}(\mathrm{Ric})$. From equations (2.6) and (2.3), we obtain

$$
\operatorname{div} \operatorname{Ric}(X)=\frac{k}{2 f^{2}}\{\Delta f d f-f d(\Delta f)(X)\} .
$$

Hence, from (2.d) and (2.1), it follows that

$$
\begin{aligned}
\operatorname{div}\left(\frac{1}{f} H^{f}\right)(X)= & \frac{1}{2 f^{2}}\left\{(k-1) d\left(|\nabla f|^{2}\right)\right. \\
& \quad-2 f d(\Delta f)+2 \lambda f d f\}+\frac{1}{f} \delta D_{B}(X, \nabla f) .
\end{aligned}
$$

But, (2.d) gives div $\operatorname{Ric}_{B}=\operatorname{div}\left(\frac{k}{f} H^{f}\right)+\operatorname{div} D_{B}$. Therefore, (2.7) and (2.8) imply that $d\left(-f \Delta f+(k-1)|\nabla f|^{2}+\lambda f^{2}\right)=0$, that is, $-f \Delta f+(k-1)|\nabla f|^{2}+\alpha f^{2}=$ $\mu$ for some constant $\mu$. Thus the first part of Proposition 2.2 is proved. For a compact Riemannian manifold $\left(F, g_{F}\right)$ of dimension $k$ with

$$
\begin{aligned}
\operatorname{Ric}_{F}(X, Y)= & g_{F}(X, Y)\left[\alpha f^{2}-f \Delta f+(k-1)|\nabla f|^{2}\right]+\beta f^{4} g_{F}(X, U) g_{F}(X, U) \\
& +\gamma f^{4}\left[g_{F}(X, U) g_{F}(Y, V)+g_{F}(Y, U) g_{F}(X, V)\right]+\delta D_{F}(X, Y),
\end{aligned}
$$

we can construct a compact super quasi-Einstein warped product $M=B \times{ }_{f} F$ by the sufficiencies of Result 2.2 . 
Now we prove the following theorem.

Theorem 2.1. Let $M=B \times_{f} F$ be a compact super quasi-Einstein warped space. If $M$ has nonpositive scalar curvature, then the warped product becomes a Riemannian product.

Proof. Equations (2.c) and (2.f) become

$$
\operatorname{div}(f \Delta f)+(k-2)|\nabla f|^{2}+\alpha f^{2}=\mu .
$$

By integrating (2.9) over $B$, we get

$$
\mu=\frac{k-2}{V(B)} \int_{B}|\nabla f|^{2}+\frac{\alpha}{V(B)} \int_{B} f^{2},
$$

where $V(B)$ denotes the volume of $B$.

1. Suppose $k \geq 3$. Let $p$ be a maximum point of $f$ on $B$. Then we have $f(p)>$ $0, \nabla f(p)=0$ and $\Delta f(p) \geq 0$. Hence, from (2.c), (2.f) and (2.10), we obtain the following:

$$
\begin{aligned}
0 \leq f(p) \Delta f(p) & =\alpha f^{2}(p)-\mu \\
& =\frac{2-k}{V(B)} \int_{B}|\nabla f|^{2}+\frac{\alpha}{V(B)} \int_{B}\left(f^{2}(p)-f^{2}\right) \leq 0 .
\end{aligned}
$$

If $\alpha<0$, then $f$ is constant.

2. Suppose $k=1,2$. Let $p$ be a minimum point of $f$ on $B$. Then we have $f(q)>0, \nabla f(q)=0$ and $\Delta f(p) \leq 0$. Hence, from (2.c), (2.f) and (2.10), we obtain the following:

$$
\begin{aligned}
0 \geq f(q) \Delta f(q) & =\alpha f^{2}(q)-\mu \\
& =\frac{2-k}{V(B)} \int_{B}|\nabla f|^{2}+\frac{\alpha}{V(B)} \int_{B}\left(f^{2}(q)-f^{2}\right) \geq 0 .
\end{aligned}
$$

If $k=1$ and $\alpha<0$, then from (2.12), $f$ is constant. If $k=2$ and $\alpha=0,(2.9)$ and (2.10) imply that $f$ is harmonic on $B$, then $f$ is constant. This completes the proof of the theorem.

\section{Example of 4-Dimensional Super Quasi-Einstein Space-Time}

Here we construct a nontrivial concrete example of a super quasi-Einstein space-time. Let us consider a Lorentzian metric $g$ on $M^{4}$ by

$$
d s^{2}=g_{i j} d x^{i} d x^{j}=-\frac{k}{r}(d t)^{2}+\frac{1}{\frac{c}{r}-4}(d r)^{2}+r^{2}(d \theta)^{2}+(r \sin \theta)^{2}(d \phi)^{2},
$$


where $i, j=1,2,3,4$ and $k, c$ are constant. Then the only nonvanishing components of Christofell symbols, the curvature tensors, and the Ricci tensors are:

$$
\begin{gathered}
\Gamma_{33}^{2}=4 r-c, \quad \Gamma_{12}^{1}=-\frac{1}{2 r}, \quad \Gamma_{22}^{2}=\frac{c}{2 r(c-4 r)}, \quad \Gamma_{32}^{3}=\Gamma_{42}^{4}=\frac{1}{r} \\
\Gamma_{33}^{2}=4 r-c, \quad \Gamma_{43}^{4}=\cot \theta, \quad \Gamma_{44}^{2}=(4 r-c)(\sin \theta)^{2}, \quad \Gamma_{44}^{3}=-\frac{\sin 2 \theta}{2} \\
R_{1221}=-\frac{k(c-3 r)}{r^{3}(c-4 r)}, \quad R_{1331}=\frac{k(c-4 r)}{2 r^{2}}, \quad R_{1441}=\frac{k(c-4 r)(\sin \theta)^{2}}{2 r^{2}} \\
R_{2332}=\frac{c}{2(4 r-c)}, \quad R_{2442}=\frac{c(\sin \theta)^{2}}{2(4 r-c)}, \quad R_{3443}=r(c-5 r)(\sin \theta)^{2} \\
R_{11}=-\frac{k}{r^{3}}, \quad R_{22}=-\frac{3}{r(c-4 r)}, \quad R_{33}=-3, \quad R_{44}=-3(\sin \theta)^{2} .
\end{gathered}
$$

From the above, it can be said that $M^{4}$ is a Lorentzian manifold of the nonvanishing scalar curvature and the scalar curvature $r_{1}=-\frac{8}{r^{2}}$. We shall now show that this manifold is $S(Q E)_{4}$.

Let us consider the associated scalars $\alpha, \beta, \gamma$ and $\delta$ and the associated tensor $D$ as follows:

$$
\alpha=-\frac{3}{r^{2}}, \quad \beta=-\frac{1}{r}, \quad \gamma=\frac{1}{r}, \quad \delta=\frac{1}{r^{2}},
$$

and

$$
\begin{aligned}
& D_{11}=0, \quad D_{22}=\frac{1}{r}, \quad D_{33}=\frac{1}{r}, \quad D_{44}=-\frac{2}{r}, \\
& D_{12}=\frac{2 \sqrt{k}}{r}, \quad D_{21}=\frac{2 \sqrt{k}}{r}, \quad D_{13}=\frac{2 \sqrt{k}}{r}, \quad D_{31}=\frac{2 \sqrt{k}}{r} \text {, } \\
& D_{14}=\frac{\sqrt{k}}{r}, \quad D_{41}=\frac{\sqrt{k}}{r}, \quad D_{23}=\frac{\sqrt{k}}{r}, \quad D_{32}=\frac{\sqrt{k}}{r}, \\
& D_{24}=\frac{1}{2 r}, \quad D_{42}=\frac{1}{2 r}, \quad D_{34}=\frac{1}{2 r}, \quad D_{43}=\frac{1}{2 r},
\end{aligned}
$$

and the 1-forms are given by

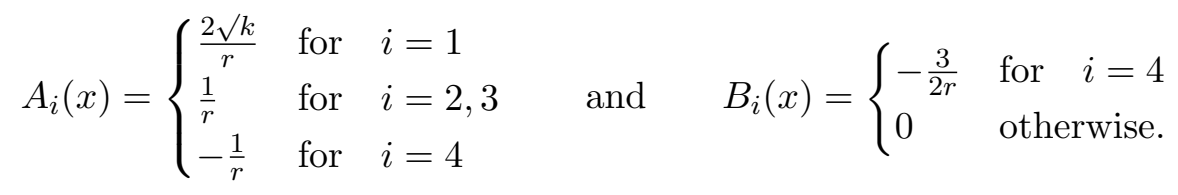

Then we have

i) $R_{11}=\alpha g_{11}+\beta A_{1} A_{1}+\gamma\left[A_{1} B_{1}+A_{1} B_{1}\right]+\delta D_{11}$, 
ii) $R_{22}=\alpha g_{22}+\beta A_{2} A_{2}+\gamma\left[A_{2} B_{2}+A_{2} B_{2}\right]+\delta D_{22}$,

iii) $R_{33}=\alpha g_{33}+\beta A_{3} A_{3}+\gamma\left[A_{3} B_{3}+A_{3} B_{3}\right]+\delta D_{44}$,

iv) $R_{44}=\alpha g_{44}+\beta A_{4} A_{4}+\gamma\left[A_{4} B_{4}+A_{4} B_{4}\right]+\delta D_{44}$.

Since all the cases other than (i)-(iv) are trivial, we can say that

$$
R_{i j}=\alpha g_{i j}+\beta A_{i} A_{j}+\gamma\left[A_{i} B_{j}+A_{j} B_{i}\right]+\delta D_{i j}, \quad i, j=1,2,3,4 .
$$

Example 3.1. Let $\left(M^{4}, g\right)$ be a Lorentzian manifold endowed with the metric given by

$$
d s^{2}=g_{i j} d x^{i} d x^{j}=-\frac{k}{r}(d t)^{2}+\frac{1}{\frac{c}{r}-4}(d r)^{2}+r^{2}(d \theta)^{2}+(r \sin \theta)^{2}(d \phi)^{2},
$$

where $i, j=1,2,3,4$ and $k, c$ are constant. Then $\left(M^{4}, g\right)$ is an $S(Q E)_{4}$ space-time with nonvanishing and nonconstant scalar curvature.

\section{Example of Warped Product on Super Quasi-Einstein Space-Time}

Here we consider the example (3.1), a 4-dimensional example of super quasiEinstein space-time endowed with the Lorentzian metric given by

$$
d s^{2}=g_{i j} d x^{i} d x^{j}=-\frac{k}{r}(d t)^{2}+\frac{1}{\frac{c}{r}-4}(d r)^{2}+r^{2}(d \theta)^{2}+(r \sin \theta)^{2}(d \phi)^{2},
$$

where $i, j=1,2,3,4$ and $k, c$ are constant. Now we have already proved that it is a super quasi-Einstein space-time with nonzero and constant scalar curvature.

Therefore the above space-time of the form $\mathbf{R} \times{ }_{f}\left(\frac{c}{4}, \infty\right) \times \mathbf{S}^{\mathbf{2}}$, where $S^{2}$ is the 2-dimensional Euclidean sphere, the warping function $f: \mathbf{R} \rightarrow(0, \infty)$ is given by $f(t)=\frac{1}{\sqrt{\frac{c}{r}-4}}, r<\frac{c}{4}$. Here $\mathbf{R}$ is the base $B$, and $F=\left(\frac{c}{4}, \infty\right) \times \mathbf{S}^{\mathbf{2}}$ is the fiber. Therefore the metric $d s_{M}^{2}=d s_{B}^{2}+f^{2} d s_{F}^{2}$, that is,

$$
d s^{2}=g_{i j} d x^{i} d x^{j}=\frac{-k}{r}(d t)^{2}+\frac{1}{\frac{c}{r}-4}\left[(d r)^{2}+\left(c r-4 r^{2}\right)\left((d \theta)^{2}+\sin ^{2} \theta(d \phi)^{2}\right)\right],
$$

is the example of a warped product on $S(Q E)_{4}$ space-time.

\section{References}

[1] A.L. Besse, Einstein Manifolds, Ergeb. Math. Grenzgeb. (3) 10, Springer-Verlag, Berlin, 1987.

[2] J.K. Beem and P. Ehrich, Global Lorentzian Geometry. Monographs and Textbooks in Pure and Applied Math. 67, Marcel Dekker, Inc., New York, 1981. 
[3] R.L. Bishop and B. O'Neill, Geometry of Slant Submnaifolds, Trans. Amer. Math. Soc. 145 (1969), 1-49.

[4] M.C. Chaki, On Super Quasi-Einstein Manifolds, Publ. Math. Debrecen 64 (2004), 481-488.

[5] M.C. Chaki and R.K. Maity, On Quasi-Einstein Manifolds, Publ. Math. Debrecen 57 (2000), 297-306 .

[6] D. Dumitru, On Quasi-Einstein Warped Products, Jordan J. Math. Stat. 5 (2012), $85-95$.

[7] M. Glogowska, On Quasi-Einstein Cartan Type Hypersurfaces, J. Geom. Phys. 58 (2008), 599-614.

[8] D. Kim, Compact Einstein warped product spaces, Trends Math. (ICMS) 52002 pp. $1-5$.

[9] D. Kim and Y. Kim, Compact Einstein Warped Product Spaces with Nonpositive Scalar Curvature, Proc. Amer. Math. Soc. 131, 2573-2576.

[10] B. O'Neill, Semi-Riemannian Geometry. With Applications to Relativity. Pure and Applied Mathematics, 103, Academic Press, Inc., New York, 1983.

[11] C. Özgür, On Some Classes of Super Quasi-Einstein Manifolds, Chaos Solitons Fractals 40 (2009), 1156-1161.

[12] B. Pal and A. Bhattacharyya, A Characterization of Warped Product on Mixed Super Quasi-Einstein Manifold, J. Dyn. Syst. Geom. Theor. 12 (2014), 29-39. 